Archives

16 | 1996

Pour une histoire comparée du vœu

\title{
Vouloir, vœu et noblesse de la volonté selon Olieu
}

\section{Olivier Boulnois}

\section{(2) OpenEdition Journals}

\section{Édition électronique}

URL : http://journals.openedition.org/ccrh/2647

DOI : $10.4000 /$ ccrh. 2647

ISSN : $1760-7906$

Éditeur

Centre de recherches historiques - EHESS

Édition imprimée

Date de publication : 16 avril 1996

ISSN : 0990-9141

\section{Référence électronique}

Olivier Boulnois, « Vouloir, vœu et noblesse de la volonté selon Olieu », Les Cahiers du Centre de Recherches Historiques [En ligne], 16 | 1996, mis en ligne le 27 février 2009, consulté le 19 avril 2019. URL : http://journals.openedition.org/ccrh/2647 ; DOI : 10.4000/ccrh.2647

Ce document a été généré automatiquement le 19 avril 2019

Article L.111-1 du Code de la propriété intellectuelle. 


\title{
Vouloir, vœu et noblesse de la volonté selon Olieu
}

\author{
Olivier Boulnois
}

1 Peut-il y avoir une théologie du vœu sans théorie de la volonté ? Il ne le semble pas, du moins dans l'exemple que je voudrais examiner. En effet, il y a dans l'instant du vœu un acte de résolution, d'engagement ou de décision primitif qui implique toute une métaphysique de la volonté.

2 Peut-il y avoir engagement sans liberté ? Sans prétendre ici répondre à cette question essentielle, je voudrais étudier une des solutions possibles, celle d'olieu, lorsqu'il affirme que seule la volonté fait de l'homme un être moral: ce qui est devenu dans l'historiographie le " primat de la volonté ", dont Olieu est le plus grand représentant ${ }^{1}$. Il n'est pas surprenant qu'une théorie très riche $\mathrm{du}$ vœu, comme celle d'Olieu, s'accompagne d'une analyse extrêmement profonde de la liberté, et d'une insistance particulière sur le primat métaphysique de la volonté. Cette étude n'a donc pas seulement un sens moral, mais aussi un sens métaphysique, puisqu'il s'agit de décider de la noblesse par excellence d'un être, l'homme.

3 Comment penser l'homme comme sujet moral ? Qu'est-ce qui en fait un sujet? Qu'est-ce qui fait la forme d'existence propre à l'homme, sa dignité distincte de toute autre dans l'échelle des créatures? Quelle est la puissance qui, en l'homme, définit le propre de l'humain? Ce sont toutes ces questions qui sont impliquées par la théorie du vœu et de la volonté.

\section{Volonté et vœu}

4 Le concept de volonté sert d'armature conceptuelle et de fondement à la théorie olivienne du vœu. En effet, selon Olieu, la possibilité de la promesse, du pacte, et donc de la confiance intersubjective reposent sur la liberté. Par conséquent, ce sont l'espérance et la société humaines qui exigent la liberté de la volonté. «C'est ce que proclament les affects d'espoir et de méfiance. Car si toutes choses sont faites nécessairement par 
l'homme, les changements de leurs résolutions (propositorum) seront eux aussi nécessaires, et l'acte de persévérer dans la même résolution proviendra, non de la liberté, mais de la nécessité. [Dans ce cas] donc, l'homme ne doit pas plus espérer dans la volonté d'un homme que dans celle d'une bête, il ne doit s'appuyer sur aucun pacte ni aucune promesse de l'homme, pas plus que dans sa société et dans son amitié, et nul ne pourra avoir aucune confiance dans sa propre résolution, puisque sa résolution ne sera pas dans sa libre puissance. Et par conséquent, toute affection (affectus) d'espoir et de sociabilité (societas), fondée dans sa résolution ou dans celle d'un autre, sera sotte et fondée sur quelque chose de faux, et donc la certitude de ce qu'on espère [Cf. Hébreux 11, 1] devra plutôt être appelée démence (amentia) que certitude $»^{2}$. La confiance de soi en soi repose sur la fermeté de la résolution, laquelle ne peut être garantie que si elle est au pouvoir d'une puissance de l'âme, la liberté, et non sujette aux aléas de la nécessité naturelle.

Mais ce principe de l'autonomie individuelle fonde aussi l'ordre public. Olieu définit la société comme une " libéralité communicative » : on ne peut pas aimer autrui comme soimême (selon le commandement du Lévitique 19,18 et de Jean 13,34 ) sans croire en autrui comme en soi-même. Sans la liberté, il ne peut y avoir de société, ni entre les hommes, ni entre l'homme et Dieu. L'absence de liberté « détruit aussi le bien que constitue toute amitié et toute société, autant envers les hommes qu'envers Dieu. En effet, toute société humaine s'appuie sur la légalité et la fidélité, et pour que l'homme soit fidèle aux autres dans les paroles, les pactes, les promesses, et même dans les faits, s'exerce une sorte de libéralité communicative.

6 L'homme est lié, non seulement par une amitié mutuelle, mais par une confiance (fiducia) et une foi (credulitas) mutuelle, de sorte que, comme l'un aime l'autre comme lui-même, il croie en l'autre comme en soi-même, et il se confie en l'autre comme il se confie en soimême $»^{3}$. Comme l'amour d'autrui se rapporte finalement à l'amour de Dieu, la confiance en autrui, fondatrice de la société, s'enracine en dernier lieu dans la confiance en Dieu. Détruire la liberté, c'est rendre cet engagement impossible. «Cela détruit aussi toute société de l'homme envers Dieu. En effet, cela supprime toute espérance des récompenses éternelles [...] toute amitié et toute grâce et toute horreur de l'inimitié divine $»^{4}$ On remarquera qu'en plaçant sur le même plan le problème de la liberté humaine et celui de la liberté divine, Olieu relie étroitement la toute-puissance divine, la liberté, et la conception de la modalité. En cela, il s'oppose à l'erreur condamnée par l'évêque de Paris Étienne Tempier en 1277, selon laquelle la liberté humaine, comme la liberté divine, est vide de sens : ce refus de la liberté est conforme à « ceux qui posent cette erreur selon laquelle, de même qu'en nous, Dieu n'aurait pas pu vouloir autre chose que ce qu'il a voulu, ni produire ses effets créés autrement qu'il ne les a créés ${ }^{5}$ Nous avons donc également besoin de penser l'homme et Dieu comme libres, tandis que c'est par le même argument que l'on nie la liberté divine et que l'on nie la liberté humaine (comme déterminée au bien que lui présente l'intellect). Par-delà le vœu, c'est la réciprocité de toute promesse qui est mise en cause, et donc la totalité de la foi chrétienne. Nier la liberté humaine, c'est détruire «tous les biens de la nature rationnelle $»^{6}$, : la moralité, le droit, les pactes mutuels, la communauté politique, la religion chrétienne. 


\section{La volonté, perfection transcendantale}

7 A quelle faculté correspond cette noblesse de la liberté ? Pour répondre à cette question, les auteurs du xiII siècle disposent de deux concepts, ceux d'intellect et de volonté. Trois possibilités sont donc ouvertes :

$81^{\circ}$ penser la liberté comme une propriété de l'intellect seul : c'est la position de Thomas d'Aquin ${ }^{7}$. Le libre arbitre, c'est la liberté de jugement, la capacité de réfléchir sur son acte de jugement. Il suppose l'appréhension du bien universel. C'est pourquoi il réside dans l'intellect et lui seul ;

$92^{\circ}$ concevoir la liberté comme une propriété commune de l'intellect et de la volonté, comme l'a fait Bonaventure ${ }^{8}$. Le modèle est clairement celui de deux causes partielles concourantes, qui produisent un effet total égal à celui de la somme des parties, et donc supérieur à celui que pourraient chacune de ses parties ;

$103^{\circ}$ analyser la liberté comme propriété de la volonté seule, même si elle organise le fonctionnement de l'intellect en général. L'idée d'une racine de la liberté dans la volonté a été développée par Henri de Gand déjà, dans sa critique de Thomas d'Aquin: pour Thomas, le sujet de la liberté est bien la volonté, mais son principe ou sa cause est l'intellect, car la volonté n'est libre que dans la mesure où elle peut se porter vers plusieurs objets, c'est-à-dire dans la mesure où elle peut présenter diverses représentations du bien, diverses déterminations de l'objet vers lequel la volonté est naturellement portée. L'alternative, et donc la liberté, est introduite par l'intellect, c'est donc lui qui est le principe de la liberté dans la volonté. Pour Henri de Gand, en revanche, la liberté a non seulement son siège, mais encore son principe dans la volonté, car elle seule a un empire sur soi-même'.

11 Henri de Gand est suivi sur ce point par Olieu ${ }^{10}$. Le modèle est clairement celui d'un principe qui se répand dans la totalité, et qui parcourt aussi la raison, mais dont l'efficace provient de la volonté seule. La liberté est la volonté. Celle-ci donne sa consistance à toute forme dérivée de liberté : la liberté du jugement ou de l'attention par exemple, n'est possible que grâce à celle de la volonté ${ }^{11}$. La liberté de l'intellect vient de celle de la volonté, et non l'inverse.

Olieu applique aussi un principe métaphysique, le principe d'éminence, selon lequel on peut attribuer la volonté à l'homme par une sorte de preuve ontologique : elle est ce pour quoi on ne peut rien penser de plus grand dans le fini : « la puissance d'aimer [...] doit être la plus haute et la plus large puissance qui puisse être pensée (quae possit excogitari), et il faut aussi qu'elle soit la plus noble, la plus simple, la plus absolue et la plus abstraite de toutes $»^{12}$. La volonté est une réalité ontologique, une perfection de l'étant: elle doit pouvoir être attribuée par excellence comme la plus haute perfection de l'homme ${ }^{13}$. On peut y voir une généralisation ontologique de ce que Bonaventure au moins appelait la «règle d'Anselme », habituellement appliquée à Dieu : «la partie la plus noble de toute contradiction logique doit être attribuée à Dieu $»^{14}$. Selon cet opérateur logique, il faut attribuer la réalité de la volonté aux natures les plus éminentes. Grâce au principe d'éminence, l'argument ontologique s'étend à toute l'échelle des perfections, y compris la volonté humaine.

13 Quelle définition justifie la noblesse de la volonté ? Selon quel critère l'attribuer plutôt qu'une autre propriété ? - Pour Olieu, la différence essentielle passe entre la nature et la 
volonté, or l'intellect est du côté de la nature : la volonté est donc une perfection plus haute que l'intellect. Les êtres les plus nobles se caractérisent ainsi par leur volonté et non par leur rationalité, et contrairement à ce que dit Thomas d'Aquin, l'âme noble se dévoile par sa volonté et non par son intellect. Ainsi, la volonté est la plus haute perfection de tout être actif. Elle a une suprême noblesse, une transcendance absolue, étant le plus haut des principes après Dieu ${ }^{15}$.

De la volonté au reste du monde créé, il y a une différence d'ordre, et donc un écart infini. D'où cette proclamation hyperbolique : la volonté est infiniment au-delà de tout autre mode d'existence, et préfère cesser d'être plutôt que de s'abaisser jusqu'à ces formes inférieures ; elle est d'un autre ordre, par rapport auquel celui-ci n'est que le néant. « $\mathrm{Si}$ l'on donnait à quelqu'un le choix d'être réduit à l'état de bête ou à celui de pur néant, chacun voudrait n'être rien, comme s'il clamait par son sens intime que tout être comparé au sien est pur néant $\aleph^{16}$. La liberté nous incite à désirer la mort ou la destruction plutôt que la réduction à l'état d'animal. L'acte de vouloir, dont le vœu est une des expressions les plus hautes, est la marque de notre dignité : hors de lui, nulle existence n'est plus désirable.

\section{Nécessité et liberté}

Comment la volonté se détache-t-elle sur le fond de la nature? La liberté sur la nécessité ? - Ici encore, Olieu rejette le déterminisme aristotélicien. L'acte de la volonté provient de sa pure spontanéité : il n'est pas déterminé par un principe naturel. La volonté est triplement indéterminée : quant à ses objets, quant à ses actes, quant à ses manières d'agir ${ }^{17}$. Elle s'auto-détermine rationnellement par elle-même, étant l'autoposition de la volonté en acte. Comme Dieu, elle est un moteur non-mû: «le premier moteur d'elle-même $»^{18}$. La volonté n'est pas la forme rationnelle que prend la causalité dans un terme de la série des causes (l'homme) : elle est à l'origine d'une nouvelle série de causes. Elle fait irruption dans l'ordre des causes comme un nouveau commencement absolu.

Pourquoi la volonté veut-elle ceci plutôt que cela? Pour l'aristotélisme, c'est parce que l'intellect lui a présenté tel objet sous l'aspect du bien. Pour Olieu, la volonté est spontanée, cause première dans son ordre, et elle n'est déterminée par rien d'autre que soi. Si la volonté était mue par une nature, elle ne serait pas libre. La liberté est principalement autodétermination de la volonté. La capacité de vouloir les contraires, comme « libertas oppositorum » est secondaire, c'est pourquoi elle peut disparaitre dans la vision béatifique sans que la nature de la liberté soit atteinte.

\section{La volonté, essence de l'homme}

(a) La volonté n'est pas seulement l'éminence de l'être en général, elle est l'excellence de la vie. Pour Olieu, la volonté est active par elle-même. Elle n'est pas mue par l'extérieur, comme semblent l'envisager les aristotéliciens qu'il rejette. La volonté est la forme de l'étant où elle réside. Comme telle, elle implique actualité, activité, vitalité ${ }^{19}$. Au rang des actes éminemment vitaux, outre l'intellect, la volonté a l'excellence. L'acte de la volonté est la vie même, l'incandescence de la vie, portée à son plus haut degré d'actualité. La vie par excellence, c'est l'acte de la volonté. « Il n'y a rien de plus noble que la liberté. $»^{20}$ 
(b) D'une manière plus paradoxale, la pensée par excellence s'avère être la volonté - et non l'intellect. Olieu se range ici dans la ligne franciscaine, qui s'oppose à la définition aristotélicienne de la liberté comme espèce noétique d'un genre plus vaste, le désir naturel ${ }^{21}$. Pour Aristote, seule la raison est puissance des contraires, et seule elle peut permettre une indifférence totale dans le choix des moyens pour atteindre notre fin naturelle, le bonheur. Pour Thomas d'Aquin, la béatitude humaine n'est que la forme rationnelle d'un désir de la nature : elle est la forme que prend le désir quand il peut s'orienter vers les objets les plus contraires que lui présente l'intellect dans une délibération rationnelle. La lignée franciscaine, au contraire, se rattache explicitement à Bernard de Clairvaux et Guillaume de Saint-Thierry. Gauthier de Bruges rappelle la formule de Bernard de Clairvaux: "Où est la volonté, là est la liberté " $^{22}$. Mais précisément, la volonté n'est pas convertible avec l'intellect : là où se trouve la raison, la liberté ne se trouve pas nécessairement, car la raison ou l'intellect peuvent être contraints à l'assentiment par une démonstration syllogistique. La raison ne donne pas la liberté à la volonté, elle n'a qu'une fonction ostensive, en proposant les termes devant lesquels la volonté doit choisirir ${ }^{23}$. Pour cette école, dont Olieu se réclame, la raison ne fonde qu'une liberté d'indifférence (libertas indifferentiae) générique devant les possibles, mais c'est la volonté, ayant la capacité de choisir un terme plutôt qu'un autre, qui la spécifie et permet la liberté parfaite (libertas perfecta) ${ }^{24}$.

Olieu va ainsi jusqu'à dénier toute rationalité à l'intellect, en une formule violente mais remarquable: "sans volonté, les hommes seraient des bêtes intellectuelles », enlever la liberté, c'est enlever " ce que nous sommes proprement, c'est-à-dire notre personnalité " ${ }^{25}$. Sans elle, nous perdons notre différence spécifique. L'humanité de l'homme ne réside donc pas dans la rationalité (Aristote) mais dans la volonté. «Rationnel» signifie bien capable des contraires, comme pour Aristote lui-même. Mais seule la volonté peut vouloir les contraires simultanément. Au moment précis où elle veut $\mathrm{A}$, elle peut encore vouloir le contraire de $\mathrm{A}$, en vertu de sa liberté foncière. «La volonté est motrice dans tout le royaume de l'âme, et toutes choses lui obéissent $»^{26}$. Lorsque l'intellect se porte vers les contraires, c'est parce que la volonté le lui ordonne et non par lui-même : il ne peut pas penser les contraires simultanément. - La plus haute noblesse consiste dans la volonté.

Olieu poursuit une ligne franciscaine qui est ici l'héritière de saint Bernard ${ }^{27}$. Mais cette thèse ne se borne pas à reprendre une anthropologie théologique. La volonté n'est pas simplement une instance plus haute, elle est le sommet à partir duquel s'inscrit la définition aristotélicienne de l'homme en son fond: la rationalité. Olieu n'oppose plus l'intellect et la volonté comme deux facultés de même rang qui se disputeraient la primauté : il voit dans la volonté la racine de l'intellectualité. L'essentiel de l'humain (c'est-à-dire du rationnel) en l'homme, c'est la liberté. L'homme en tant qu'homme est seulement volonté : la racine de la personnalité humaine est la liberté28. Parti d'une réflexion sur les fondements de l'engagement humain, Scot arrive à une définition de l'homme par sa puissance la plus intime, la volonté : une définition extrême, paradoxale, et finalement non-anthropologique. 


\section{NOTES}

1. Duns Scot étant sur ce point un disciple fidèle, malgré quelques différences caractéristiques mais secondaires, évoquées dans O. Boulnois, «La base et le sommet, la noblesse de la volonté selon Duns Scot ", à paraître dans les Actes du Congrès international de la Société internationale d'études de la philosophie médiévale, Ottawa, août 1992.

2. In II Sententiarum, "An in homine sit liberum arbitrium ?", éd. Jansen, Quaracchi, II, 1924, q. 57, p. 322.

3. In II Sententiarum, p. 337.

4. In II Sententiarum, p. 337-338.

5. In Sententiarum, p. 338.

6. Olivi, In II Sententiarum, q. 57, p. 316.

7. Contra Gentiles II, 48, §.1243: «Horum igitur haec sola libere iudicant quaecumque in iudicando seipsa movent. Nulla autem potentia iudicans seipsam ad kudicandum movet nisi supra actum suum refelctatur: oportet enim, si se ad iudicandum agit, quod suum iudicium cognoscat. Quod quidem solius intellectus est. Sunt igitur animalia irrationalia quodammodo liberi quidem motus sive actionis, non autem liberi iudicii [...] intellectualia non solum actionis, sed etiam liberi iudicii, quod est liberum arbitrium habere ».

8. Bonaventure, Sentences II, d. 25, p. 1, a. 1, q. 3 : «Liberum enim arbitrium, sive nominet potentiam sive habitum, nominat ipsam potentiam ut dominam vel dominium illius potentiae [... ]. Ad hoc igitur ut illud dominium sit in anima, necesse est quod habeat et rationem et voluntatem. Nam si rationem tantum haberet et non voluntatem per quam moveret, posset se super actum suum reflectere, sed non posset movere vel imperare. [...] Sicut igitur ex concursu virtutis duorum hominum resultat quaedam facultas ad portandum unum lapidem, quem non posset ferre alter eorum [...] sic ex concursu rationis et voluntatis resultat quaedam libertas sive quoddam dominium ad aliquid faciendum et disponendum ». Godefroid de Fontaines, Quodlibet VI, q. 10, III, p. 206-209.

9. Henri de Gand, Quodlibet IX, q. 6, Venise, 1613 ; II, 88 a, : « Unde quod dicunt aliqui, quod radix libertatis sicut causa est ratio sive intellectus, licet subiectum eius sit voluntas, ut ex hoc solo voluntas libera possit ad diversa ferri, quia ratio potest habere diversas conceptiones boni ; absit hoc, quod alibi ostendimus : non potest stare, immo voluntas est subiectum libertatis et radix prima, a qua per participationem ad imperium, et impressionem eius invenitur in actibus rationis et alium virium. "

10. Commentarius ordinarius in II librum Sententiarum, distinctio 24, art. 2: «Quaeritur utrum liberum arbitrium sit una potentia vel plures ", Bibl. Univ., Padoue, Cod. 637, Fo $156 \mathrm{~cd}$, ed. F. Simoncioli, Il problema della liberta umana in Pier di Giovanni Olivi e Pietro de Trabibus, Milano, 1956 : «Et ideo libertas arbitrii totaliter est ipsa voluntas quantum ad radicem et consistentiam suam. Operatio autem sua actualis, ut disctum est, ordinem habet ad intellectum, et aliquando ipsum includit concomitative et pro quanto actus liber est cum deliberatione et animadversione ac iudicio rationis. "

11. Olieu, Quaestiones in Secundum Sententiarum, éd. Jansen III, 26 : « Potentia cognitiva non potest se ipsam movere et convertere ad sua obiecta nec ad suos actus cognitivos, quia solius potentiae voluntatis est movere se ipsam et alias potentias. »

12. Quaestiones In II Sententiarum, p. 331.

13. W. Hoeres, Der Wille als reine Vollkommenheit nach Duns Scotus, München, 1962, p. 25 sq. 
14. Bonaventure, Collationes in decem praeceptis III, 27, Opera omnia V, Quaracchi, 1891, p. 519 ; Olivi, Quaestiones de incarnatione et redemptione. Quaestiones de virtutibus, q. 1, éd. A. Emmen, E. Stadter, Grottaferrata, 1981, p.12. Le motif de la généralisation scotiste est le principe aristotélicien de plénitude : « Illud enim est ponendum in natura, quod melius est, si sit possibile, ex secundo de Gen[eratione et corruptione, 336 b, 27-28]. Natura enim semper desiderat quod melius est ». Chez Anselme, Monologion, ch.4, le principe s'applique principalement à Dieu: "Si quis intendat rerum naturas, velit nolit sentit non eas omnes contineri una dignitatis paritate, sed quasdam earum distingui graduum imparitate. [...] Cum igitur naturarum aliae aliis negari non possint meliores, nihilominus persuadet ratio aliquam in eis sic supereminere, ut non habeat se superiorem. "

15. Selon les mots de P.J. Olieu, : «transcendit omne creatum », Quaestiones in II Sententiarum, ed. Simoncioli, Il problema della liberta umana in Pietro di Giovanni Olivi e Pietro de Trabibus, Milan, 1956, p. 187. E. Stadter, Psychologie und Metaphysik der menschlichen Freiheit. Die ideengeschichtliche Entwicklung zwischen Bonaventura und Duns Scotus, 1971, München-Paderborn-Wien, p. 290.

16. «Sentimus cor nostrum quasi in infinitum excedere omnem alium modum existendi. Unde si cui daretur optio in quod minus vellet redigi, scilicet, in unum animal, aut in purum nihil tantum : unusquisque vellet esse nihil, ac si intimo sensu clamet quod omne esse comparatum ad suum est quasi purum nihil", Quaestiones in Secundum Librum Sententiarum, ed. B. Jansen, Quaracchi, 1924, II, q. 57, p. 334, p. 335 : «defectum usus liberi arbitrii, qualis est in stultis, si deberet esse perpetuus, ita horreremus sicut annihilationem. »

17. Quaestiones in Secundum Sententiarum, q. 58 resp. ; II, p. 326-328.

18. "Necesse est quod liberum arbitrium habeat rationem primi motoris", Quaestiones in Secundum Sententiarum, q. 58 resp.; II, 411 ; «Ad hoc enim quod actum libere producat exigitur quod actus ille exeat ab ea secundum aspectum dominativum, ita quod voluntas exeat in illum tamquam a se mota et non tamquam ab alio applicata et quais necessitata », 58 ad 9 ; II, p. 429.

19. Olivi, Quaestiones, III, q. 54 ad 1, Jansen II, p. 274 : « Multas habent conditiones nobiles [...], scilicet summam vitalitatem, seu vivacitas vel vitae actualitatis». Commenté par E. Stadter, op. cit., p. 294.

20. Olieu, Quaestiones in Secundum Librum Sententiarum "Nihil autem est nobilius libertate ", Jansen II, p. 249.

21. Thomas d'Aquin, Somme théologique II-II, q. 17, a. 1 ad 2 : la liberté a pour lieu la volonté, mais pour cause la raison.

22. De gratia et libero arbitrio, ch. 1, §. 2. Sur cette tradition: J.M. Déchanet, "Amor ipse intellectus est", Revue du moyen-âge latin, 1, 1945, p. 350-374 et la conclusion de la page 373 : «nous nous trouvons en présence d'une véritable théologie de l'amour-intellection ». L'expression se trouve chez Guillaume de Saint-Thierry dans le Miroir de la foi, ch. 10, §. 97, Sources Chrétiennes, p. 301, éd. et trad. J.M. Déchanet. L'éditeur lui donne pour sources Bernard, Grégoire le Grand, et même Plotin, Ennéade VI, 7, 35, p. 24-25.

23. Gauthier de Bruges, Quaestiones disputatae, Louvain, ed. E. Longpré, 1928, q. 5 : «Quaeritur unde voluntas habeat quod sit libera », arg. II. 1 : «Ubi voluntas, ibi libertas; sed non ubi ratio, semper ibi libertas, quia ratio vel intellectus demonstratione syllogistica cogi potest; ergo voluntas a se, non a ratione, est libera ». Resp. ad II. 1 : «Primum in contrarium concede sic : quod ratio, ut est potentia, non dat libertatem voluntati effective nec formaliter, sed tantum ostensive, quia proponit sibi duo vel plura, de quibus unum est praeoptandum. »

24. Gauthier de Bruges, op. cit., II ad 14, p. 53, [voluntas] «habet libertatem indifferentiae a ratione, ut est nomen essentiae, sed libertatem praeferendi unum alii a seipsa voluntate». Commenté par E. Stadter, op. cit., p. 314. Quatre siècles avant Descartes, l'opposition entre liberté de perfection et liberté d'indifférence est plusieurs fois reprise : «omnis autem ratio et secundum ipsam rationalis potentia habet indifferentiam ad opposita, quae est libertas aliqua, quia non est ad unum per aliquem sui actum vel differentiam arctata, sed non est libertas perfecta, quia non potest 
alterum oppositorum praeoptare" (Resp. p. 51) ; "Ad libertatem tria concurrunt, scilicet quod duo vel plures diversa proponantur, et quod in eligente indifferentia quaedam ad illa habeatur, et quod unum altero vel aliis praeoptetur. Primum habet voluntas a ratione ut est potentia conferens et proponens voluntati quid volendum ; secundum habet a ratione ut est essentia, quae est in eo quod ratio habet indifferentiam ad utrumlibet horum; tertium habet a seipsa, scilicet voluntate, scilicet unius illorum praeoptationem, et quia in hoc consummatur libertas, ideo proprie et quasi formaliter a se, vel per illud quod habet in se, est libera. Sed ratio, et potentia, ostendi sibi libertatis materiam, sed ratio, ut essentia, dat sibi quamdam quasi generis indifferentiam ", p. 52 : je souligne.

25. Quaestiones in II Sententiarum, q. 57, II, p. 338. Texte cité par V. Doucet, « De operibus mss Fr. Petri Ioannis Olivi in Bibl. Univ. Patavini asservatis ", Archivum franciscanum historicum, 28, 1935, 179, n. 9: «liberum arbitrium, quantum ad hoc quod dicit facultatem liberam et dominium habentem super totam animam, essentialiter est voluntas rationalis [...] libertas essentialiter est voluntas, et libere consentire est actus eius immediatus et totalis, ad quem nihil facit intellectus nisi per accidens ".

26. Anselme, De conceptu virginali, ch. 5 : «Voluntas est motor in toto regno animae, et omnia oboediunt sibi ». Scot, Opus Oxoniense, II, d. 42, q. 4, n. 1-2.

27. O. Boulnois, postface de Pic de la Mirandole, Euvres philosophiques, Paris, 1993.

28. Quaestiones in Secundum Sententiarum, q. 51 resp., Jansen II, p. 121 : « Non enim potest esse aliquid radix personalis subistentiae nisi illud quod est super seipsum plene rediens et in seipso liberrime consistens"; q.52, dicendum, II, p. 200: [Personalitas] "est idem quod per se existentia dominativa et libera et in se ipsam possessive vel reflexibilis, id est, se ipsam cum quadam libera reflexione possidens. »

\section{AUTEUR}

\section{OLIVIER BOULNOIS}

EPHE, $\mathrm{V}^{\mathrm{e}}$ section 